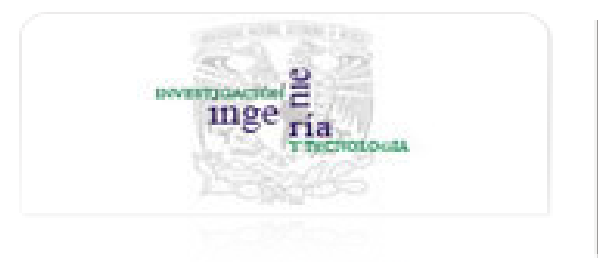

Ingeniería. Investigación y Tecnología

ISSN: 1405-7743

iit.revista@gmail.com

Universidad Nacional Autónoma de México

México

Gardea Villegas, $\mathrm{H}$.

Dos propuestas para el proyecto del bombeo de fluidos no newtonianos. Caso de los lodos residuales de plantas de tratamiento de aguas negras

Ingeniería. Investigación y Tecnología, vol. IX, núm. 2, abril-junio, 2008, pp. 87-97

Universidad Nacional Autónoma de México

Distrito Federal, México

Disponible en: http://www.redalyc.org/articulo.oa?id=40490201

- Cómo citar el artículo

- Número completo

- Más información del artículo

Página de la revista en redalyc.org

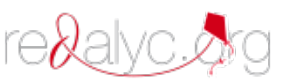

Sistema de Información Científica

Red de Revistas Científicas de América Latina, el Caribe, España y Portugal

Proyecto académico sin fines de lucro, desarrollado bajo la iniciativa de acceso abierto 
INGENIERÍA INVESTIGACIÓN Y TECNOLOGÍA IX. 2.87-97,2008

(ARTíCULO ARDITRAdO)

EN MÉXICO

INGE RÍA

Y EN El MUNdO

\title{
DOS PROPUESTAS PARA El PROYECTO del bOMbeO de Fluidos NO newtonianos. Caso de los lodos residuales de plantas de TRATAMIENTO dE AGUAS NEGRAS
}

\section{TWO PROPOSALS FOR PUMPING CALCULATIONS OF NON-NEWTONIAN FluidS, WATER TREATMENT PlANTS disPOSAl SludGES CASE}

\author{
H. Gardea-Villegas \\ Facultad de Ingeniería UNAM, México \\ E-mail: gardea@servidor.unam.mx
}

(Recibido: novembre de 2006; aceptado noviembre de 2007)

\section{Resumen}

Se presentan dos propuestas para el cálculo del bombeo de fluidos no newtonianos, con aplicación directa al caso de los lodos residuales de plantas de tratamiento de aguas negras. Las propuestas se apoyan en métodos sugeridos por Levenspiel (1986) aplicables únicamente a fluidos Bingham plásticos y pseudoplásticos simples en cualquier tipo de régimen. Ante el hecho señalado por este autor, de que aun no se ha desarrollado la gráfica que relacione el factor de fricción con el número de Reynolds para cualquier material plástico, no es posible calcular directamente el Bingham pseudoplástico, que es precisamente el tipo al que pertenecen los lodos residuales, objetivo de este trabajo, el mencionado investigador sugiere un procedimiento que está sujeto al criterio del proyectista y por ello, habrá apreciaciones personales con las desventajas que esto conlleva. Por esta razón se propone un método de cálculo para lodos residuales, extensivo desde luego a otro tipo de fluidos no newtonianos, que si bien, se apoya en el criterio original de Levenspiel, elimina o disminuye mucho la parte subjetiva del proyecto, llegándose a resultados más consistentes. Esto es parte del trabajo de doctorado que realiza el autor bajo la dirección del Dr. Rafael B. Carmona Paredes en la Facultad de Ingeniería de la UNAM.

Descriptores: Fluidos no newtonianos, lodos residuales, algoritmos para el cálculo del bombeo, Bingham pseudoplásticos, plantas de tratamiento de aguas negras, régimen de flujo.

\section{Abstract}

This paper presents two ways to calculate the pumping power of non Newtonian fluids and especially yield pseudoplastics which are the kind of disposal fluids from Water Treatment Plants. Fluids called sludges. The proposals included here, are based in methods suggested by Levenspiel (1986) applicable to determine the performance of Bingham plastics and pseudoplastic fluids using a graphical approximation of the rheological behavior of these materials. This approach has the advantage that is appropriate to any kind of regime. Otherwise, Levenspiel underlines, that there is not yet a chart who relates the roughness coefficient with the Reynolds number for general plastics, so it is not possible by now to calculate the yield pseudoplastic fluid. Its calculation is the aim of this study. Levenspiel proposes an approach subject to the assessment of the project manager, and will therefore entail personal observations, with the limitations that this can cause. The results obtained by both propositions, are very 
similar. This is part of a doctorate study done by the author under the direction of Dr. Rafael B. Carmona in the Faculty of Engineering of the National Autonomous University of Mexico.

Keywords: Non-Newtonian fluids, sludges, algorithms to calculate pumping, yield pseudoplastic, water treatment plants, fluid regime.

\section{Introducción}

Las plantas de tratamiento de aguas negras, producen lodos residuales que aun pueden ser aprovechados si se someten a ciertos procesos químicos. Por otra parte, el procesamiento de estos lodos requiere amplias zonas expuestas a la atmósfera y por ello se presenta la necesidad de transportarlos de la planta a terrenos adecuados que normalmente se encuentran fuera de las ciudades.

El problema es especialmente serio en las grandes zonas habitadas como es el caso de la Ciudad de México en la que las distancias a las que deben llevarse dichos lodos, pueden ser muy grandes.

El transporte puede hacerse, desde luego en camiones cisterna o con otros procedimientos más o menos costosos y por ello, se pretende utilizar el bombeo como la solución más ventajosa y segura. Sin embargo, el bombeo implica algunas dificultades porque los lodos residuales son materiales con características peculiares, ya que no son fluidos newtonianos y esto implica la necesidad de aplicar consideraciones especiales en los proyectos que diferencian notablemente el transporte de lodos de los casos en que se maneja agua clara, que es a lo que está acostumbrado el ingeniero civil.

\section{Ecuación general del comportamiento de los fluidos}

Cuando se trata de estudiar el movimiento de líquidos por una tubería de sección circular en régimen laminar, lo más apropiado es usar expresiones en las que se usen coordenadas cilíndricas. Por ello, el modelo de Herschel-Bulkley es sin duda el más apropiado (Slatter, 2004) y de acuerdo con la figura 1 está descrito por la siguiente expresión:

$$
\tau=\tau_{Y}+\mathrm{K}(-\mathrm{du} / \mathrm{dr})
$$

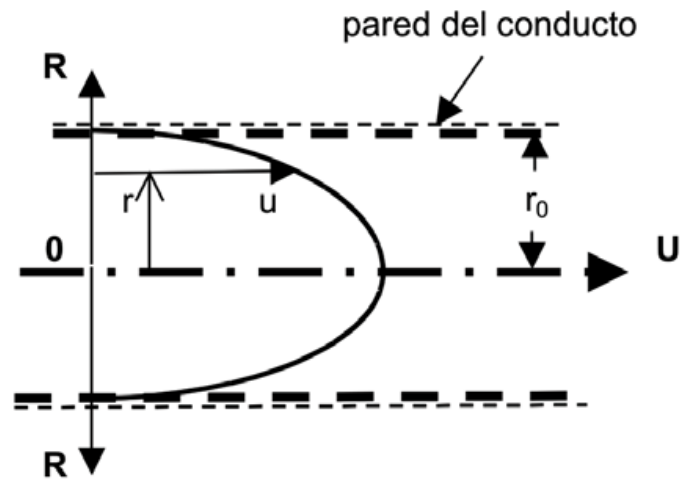

Figura 1

En la figura 2 (Slatter, 1997) se indica la clasificación de los principales tipos de fluido y de acuerdo con la ecuación anterior, en el cuadro 1 se indican los parámetros generales que caracterizan a dichos fluidos.

El significado de las variables señaladas es el siguiente:

$\tau[\mathrm{Pa}]$, es el esfuerzo cortante experimentado por una partícula que se encuentra a una distancia $\mathbf{r}$ del eje de la tubería y está animada por una velocidad $\mathbf{u}$. 
$\tau_{y}[\mathrm{~Pa}]$, es el esfuerzo de fluencia necesario para que el fluido entre en movimiento (Figura 2).

$\mathbf{K}\left[\mathrm{Ns}^{n} / \mathrm{m}^{2}\right.$ o Pa.sn], es el llamado índice de consistencia del fluido, (Slatter, 1997), también llamado coeficiente de viscosidad plástica (constante), (Lester, 1994).

n es el índice de comportamiento del fluido (Honey, 2000 y Slatter, 1997), parámetro constante y característico de cada líquido. A los fluidos cuyo exponente $n \neq 1$, se les llama también fluidos exponenciales.

\begin{tabular}{|c|c|c|}
\hline \multicolumn{3}{|c|}{ NO NEWTONIANOS } \\
(ec. 1) \\
\hline & $\tau_{y}=0$ & $\tau_{y}>0$ \\
\hline $\mathrm{n}<1$ & Pseudoplástico & Bingham pseudoplástico \\
\hline $\mathrm{n}>1$ & Dilatante & Bingham dilatante \\
\hline $\mathrm{n}=1$ & $*$ & Bingham plástico \\
\hline
\end{tabular}

Cuadro 1

* Newtonianos, sujetos a la ley: $\tau=\mathrm{K}(-\mathrm{du} / \mathrm{dr})$, que no es otra cosa que la conocida ley de viscosidad de Newton. En este caso $\mathrm{K}$ es el coeficiente de viscosidad dinámica del fluido, indicado generalmente con la letra griega $\mu[\mathrm{cp}, \mathrm{kg} /(\mathrm{ms})]$.

Las investigaciones han demostrado que los lodos residuales provenientes de las plantas de tratamiento de aguas negras son del tipo Bingham pseudoplástico, por lo que se hará referencia precisamente a este caso.

\section{Conveniencia de usar régimen laminar con fluidos no newtonianos}

Desde luego, si se observa la fórmula de Poiseuille: $f=64 / R e$, es evidente que en régimen laminar, tratándose de fluidos newtonianos, el aumento de la velocidad, que implica un incremento en el número de Reynolds, produce una disminución del factor de rugosidad $\mathrm{f}$, mientras que, como es sabido, en la zona turbulenta en la que f es constante para un conducto específico, la pérdida por fricción aumenta en función del cuadrado de la velocidad, como señalan todas las fórmulas de fricción y por consiguiente las pérdidas se incrementan en forma importante con el aumento de la velocidad. En el caso de los fluidos no newtonianos, este último efecto es aún más notable como lo demuestra Slatter (1997) en los experimentos que realizó y que se indican en la figura 3.

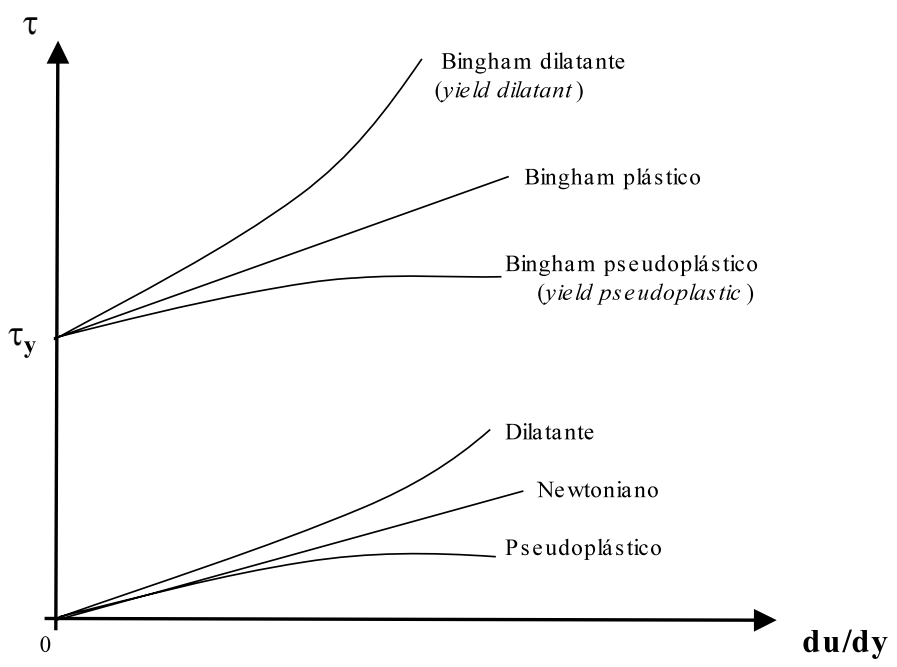

Figura 2 


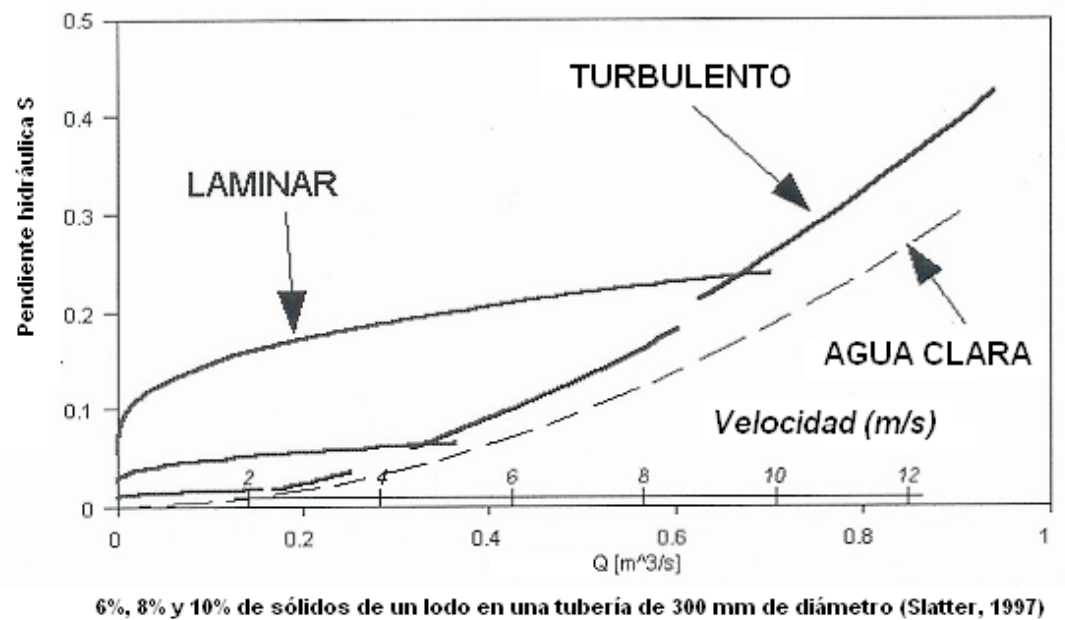

Figura 3

En esta figura se observa que si bien los incrementos de la pendiente hidráulica $S$ van disminuyendo al aumentar la velocidad (el número de Reynolds). Al pasar a la zona turbulenta se presenta una elevación brusca de la pendiente hidráulica $\mathrm{S}$ y por consiguiente de la pérdida por fricción, algo que no sucede para el agua clara, como se puede ver en la misma figura.

Posteriormente, Slatter y Wasp (2000), relacionaron experimentalmente el esfuerzo de fluencia $\tau_{y}$ de un fluido con la velocidad del mismo en el paso del régimen laminar al turbulento en tuberías largas y obtuvieron una fórmula para determinar dicha velocidad que denominaron velocidad crítica $\mathrm{V}_{\mathrm{cs} \& w}$. Esta fórmula es la siguiente:

$$
\mathrm{V}_{\mathrm{cS \& W}}=26 \sqrt{\frac{\tau_{y}}{}}
$$

Ampliando sus investigaciones, Slatter (2004) concluye, que la turbulencia no se presentará a velocidades "reales, menores de $3 \mathrm{~m} / \mathrm{s}$ aproximadamente" y que si el esfuerzo de fluencia es mayor de $13 \mathrm{~Pa}$ el régimen es laminar si no se excede dicha velocidad de $3 \mathrm{~m} / \mathrm{s}$.
Por su parte Shook y Roco (1991) ya habían señalado que la mezcla rápida de las partículas del líquido consume una cantidad de energía mucho mayor que la producida por el simple deslizamiento entre las capas del fluido, característica de un régimen laminar y que este incremento en el consumo de energía va acompañado también de un aumento del esfuerzo cortante en las paredes del conducto y por consiguiente de mayor pérdida de energía en la conducción.

Estos argumentos apoyan la recomendación de los investigadores de que el régimen apropiado para fluidos no newtonianos debe ser laminar y de acuerdo con la figura 3, el más conveniente es el que se encuentre cerca del límite de principio de la zona turbulenta.

\section{Procedimiento de cálculo de Levenspiel (1986), para la determinación del factor de fricción con lodos residuales (Bingham pseudoplásticos)}

Este investigador señala que aún no se ha resuelto el problema específico del cálculo de la caída de presión en una tubería que transporta un fluido Bingham pseudoplástico, pero sí plantea un 
procedimiento para calcular el Bingham plástico $\left(\tau_{\mathrm{y}}>0\right.$ y $\left.n=1\right)$ y el pseudoplástico simple $\left(\tau_{\mathrm{y}}=0\right.$ y $n<1)$. La solución que propone Levenspiel consiste en descomponer el Bingham pseudoplástico en dos, a saber: un Bingham plástico, haciendo $\mathrm{n}=1$ en la expresión de Herschel-Bulkley y un pseudoplástico simple, haciendo $\tau_{y}=0$ en la misma ecuación. Posteriormente, calcular ambos líquidos e interpolar los resultados en alguna forma, dice el investigador, ya que el valor correcto del Bingham pseudoplástico tendrá necesariamente que encontrarse entre el Bingham plástico y el pseudoplástico simple, que son los dos fluidos en los que se ha descompuesto el original. Más abajo se harán dos propuestas de cálculo que pretenden eliminar o al menos reducir en lo posible, el factor de apreciación de cada proyectista, pero antes se describe el método de cálculo de Levenspiel para ambos casos.

\section{Procedimiento de Levenspiel para el cálculo del factor de fricción con fluidos tipo Bingham plástico $\left(\tau_{y}>0, n=1\right)$}

1. Determinar experimentalmente los parámetros: $\tau_{y}, \gamma, \rho$ y $\mathrm{K}$

2. Calcular el número de Reynolds de la mezcla:

$$
\operatorname{Re} \frac{\rho V D}{K} ;
$$

(D, diámetro de la tubería)

3. Calcular el número de Hedstrom:

$$
H e \frac{D^{2} \tau_{y} \rho}{K^{2}}
$$

4. Determinar el factor de fricción de Fanning $\mathrm{f}_{F}$, con los parámetros Re y He en la gráfica de Hedstrom adaptada por Levenspiel (Figura 4) y a partir de este valor, obtener el factor $f$ de Darcy $\left(f=4 f_{F}\right)$.

En este caso el método para realizar el cálculo puede describirse en la siguiente forma:

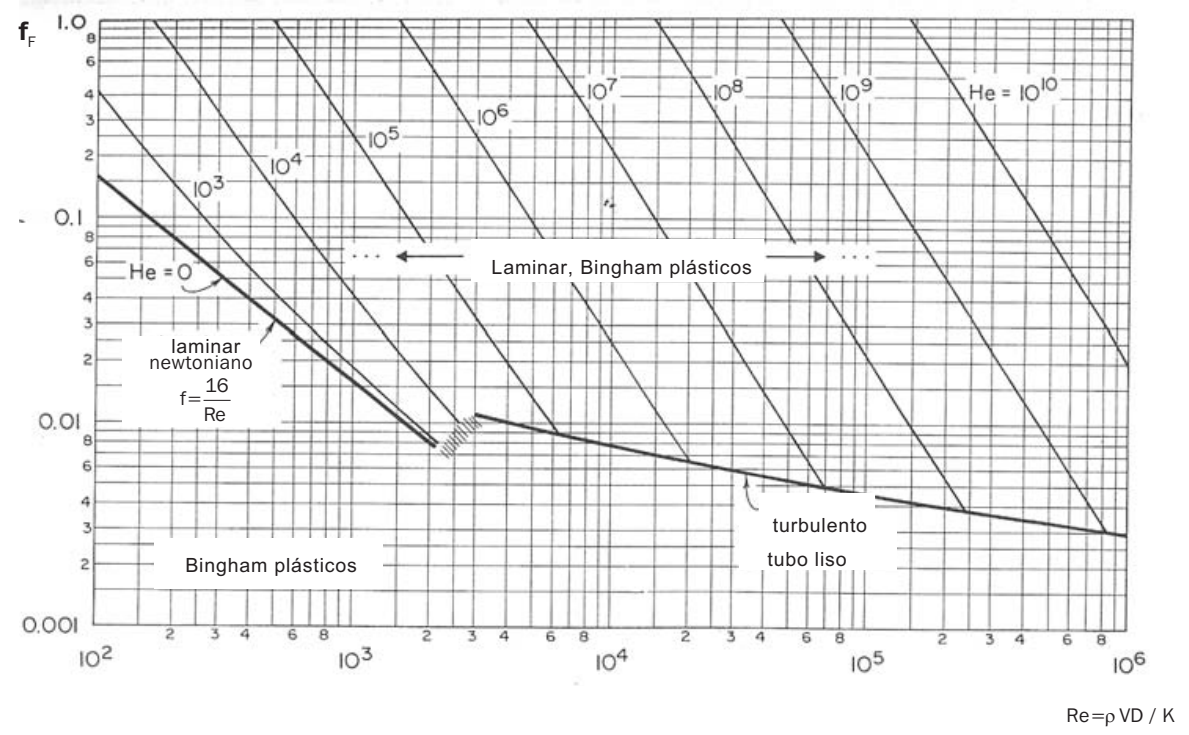

Figura 4. (Levenspiel, 1986) 
5. Calcular la carga dinámica de la bomba y la potencia necesaria:

$$
P \quad Q H / \eta_{b} ;\left[k g_{f} m / s\right]
$$

$\left(\eta_{b}\right.$, eficiencia de la bomba)

Procedimiento de Levenspiel para el cálculo del factor de fricción con fluidos pseudoplásticos simples $\left(\tau_{y}=0, n<1\right)$

La ecuación reológica para los fluidos pseudoplásticos simples $(n<1)$, según el modelo de Herschel-Bulkley es:

$$
\tau \quad K(d u / d r)^{n}
$$

El método de cálculo puede describirse en la siguiente forma:

$1^{\circ}$ Calcular el número de Reynolds generalizado, que el investigador define con la siguiente expresión:

$$
\operatorname{Re}_{\operatorname{gen}} \frac{D^{n} V^{2}{ }_{m}}{8^{n}{ }^{1} K} \frac{4 n}{3 n 1}{ }^{n}
$$

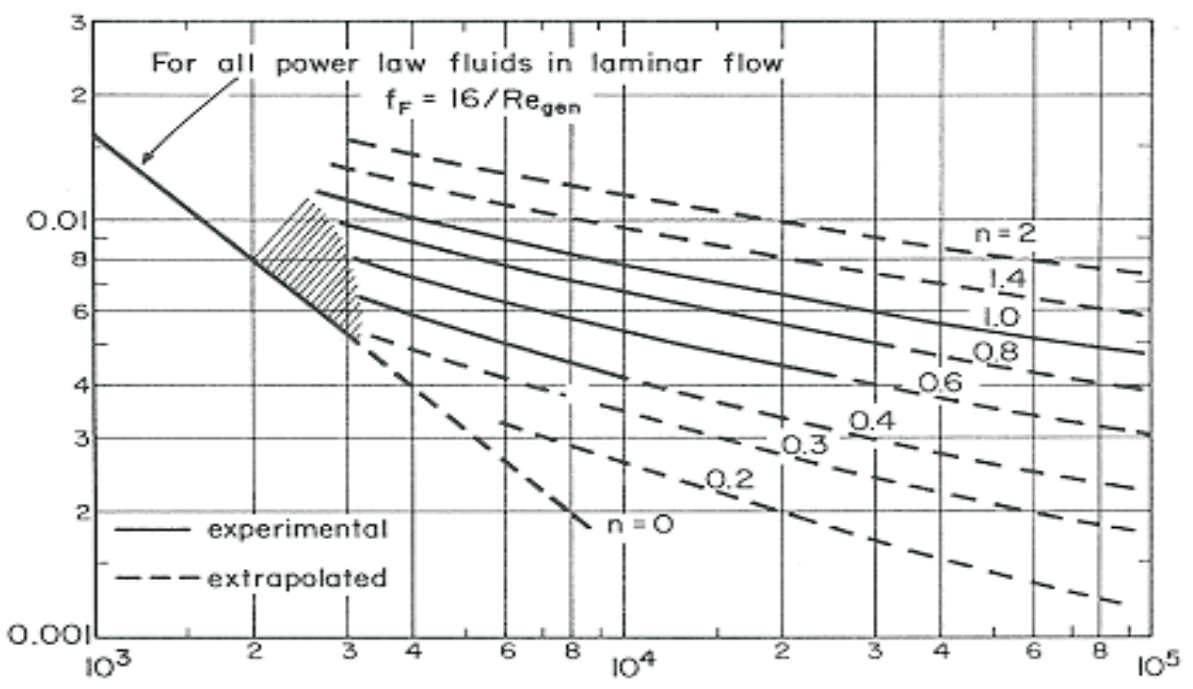

$2^{\circ}$ Con $\operatorname{Re}_{\text {gen }}$ y $\mathrm{n}$ obtener el factor de fricción de Fanning $f_{F}$ con la gráfica obtenida por Dodge y Metzner adaptada por Levenspiel, que se presenta en la figura 5 . Recuérdese que $f_{F}=f / 4$, siendo $f$ el factor de fricción de la fórmula de Darcy.

$3^{\circ}$ Calcular las pérdidas con la fórmula de Darcy y la carga dinámica de la bomba.

$4^{\circ}$ Calcular la potencia de la bomba.

\section{Propuestas para el cálculo de un fluido Bingham pseudoplástico}

La idea que se plantea en este artículo consiste en adaptar el fluido original a otro equivalente para el que habrá una de las siguientes dos opciones:

Propuesta 1.

Adaptar el fluido real a un Bingham plástico, haciendo $\mathrm{n}=1$

Propuesta 2.

Adaptar el fluido real a un pseudoplástico simple, haciendo $\tau_{\mathrm{y}}=0$ 
Como se ha señalado, este tipo de fluidos sí se puede calcular. Entonces el procedimiento que se propone es hacer el cálculo de cualquiera de las dos opciones y atribuir su resultado al fluido real.

Enseguida, se presenta una aplicación de ambas propuestas, con base en el siguiente ejemplo. Se utilizan los datos de un lodo Bingham pseudoplástico manejado por Slatter (1997).

\section{Problema}

Calcular la potencia de bombeo para el proyecto cuyos datos se indican, utilizando un lodo residual que es un fluido tipo Bingham pseudoplástico. Los datos son los siguientes:

$L=12,000.00$ m; eficiencia de la bomba: $\eta_{b}=$ $0.68 ; \mathrm{H}_{\mathrm{ET}}=80.00 \mathrm{~m}$ (desnivel estático); $\mathrm{D}=$ $0.2032 \mathrm{~m} ; \mathrm{V}=1.54 \mathrm{~m} / \mathrm{s} ;\left(\mathrm{Q}=0.050 \mathrm{~m}^{3} / \mathrm{s}\right) ; \rho=$ $1,008.00 \mathrm{Kg} / \mathrm{m}^{3} ; \mathrm{K}=0.3660$ Pa. $\mathrm{s}^{n} ; \mathrm{n}=0.664$; $\tau_{\mathrm{y}}=12.00 \mathrm{~Pa}$.

\section{Propuesta 1}

Adaptación a un Bingham plástico
De acuerdo con la ecuación general, la ley reológica que describe al fluido, es la siguiente:

$$
\tau=12+0.3660(-d u / d r)^{0.664}
$$

En la figura 6 se representa este fluido, graficado con el programa Maple 9.5

\section{Cálculo siguiendo el procedimiento de Levenspiel para el Bingham plástico}

El Bingham plástico equivalente, que se indica en la figura 7 con línea punteada, se obtuvo ajustando la curva real de la figura 6 . En la figura 7 se representa con línea también el fluido real.

En la misma figura 7 se indican las ecuaciones de ambas curvas y con los parámetros señalados, se obtienen los siguientes valores para Re y He:

Número de Reynolds:

$$
\operatorname{Re} \frac{\rho V D}{K} \quad 2,937.65
$$

Número de Hedstrom:

$$
H e \frac{D^{2} \tau_{y} \rho}{K^{2}} \quad 43,217.15
$$

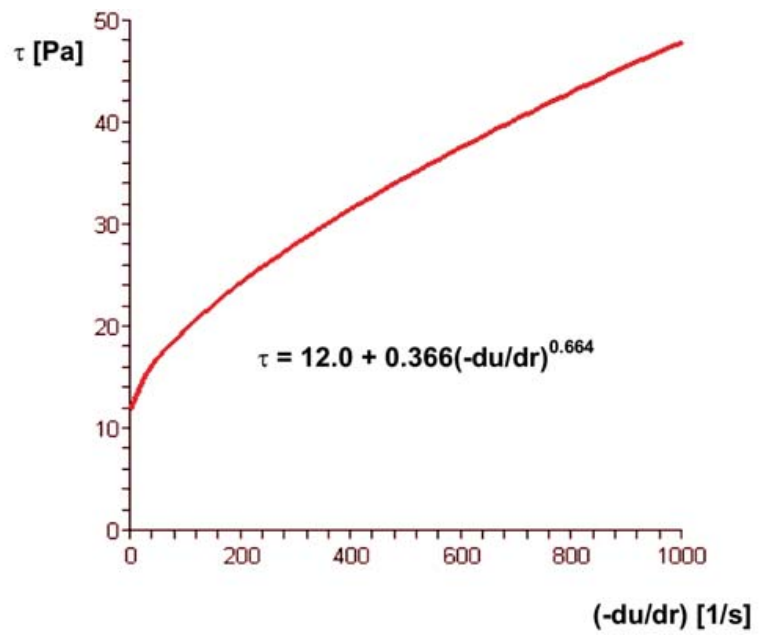

Figura 6 
Con Re y He, en la gráfica de la figura 4 se lee $f_{F}=$ 0.016 , equivalente $a f_{\text {Darcy }}=f=4 f_{F}=0.064$. Por lo que la pérdida por fricción es:

$$
\Sigma \mathrm{h}_{f}=\mathrm{fL} / \mathrm{D}\left(\mathrm{V}^{2} / 2 \mathrm{~g}\right)=457.94 \mathrm{~m}
$$

y la carga dinámica del bombeo:

$$
\mathrm{H}=\mathrm{H}_{E T}+\Sigma \mathrm{h}+\mathrm{V}^{2} / 2 \mathrm{~g}=538.06 \mathrm{~m}
$$

Entonces, la potencia de bombeo buscada es:

$$
\mathrm{P}_{(H P)}=\gamma \mathrm{QH}\left(76.04 \eta_{b}\right)=524.45 \mathrm{HP}
$$

\section{Propuesta 2}

Adaptación a un pseudoplástico simple

En la figura 8 se indica el ajuste de la curva del lodo real a un pseudoplástico simple equivalente, este último representado con línea de puntos. En la figura también se anotan las ecuaciones de ambas curvas.

\section{Cálculo siguiendo el procedimiento de Levenspiel para un pseudoplástico simple}

El número de Reynolds generalizado de acuerdo con los datos del ejemplo y los parámetros obtenidos e indicados en la figura 8 para el pseudoplástico equivalente, tiene el valor

$$
\operatorname{Re}_{\text {gen }} \frac{D^{n} V^{2} n}{8^{n}{ }^{1} K} \frac{4 n}{3 n 1}^{n} \quad 988.81<2200 \text { (laminar) }
$$

(En la figura 5 se observa claramente que se trata de un régimen laminar)

En estas condiciones, es válida la fórmula de Poiseuille: $f=64 / \operatorname{Re}=0.0647$. La carga dinámica de bombeo es entonces

$$
H=H_{E T}+f L / D v^{2} / 2 g+v^{2} / 2 g=542.15 m
$$

Y la potencia necesaria

$\mathrm{P}=\gamma \mathrm{QH} /\left(76.04 \eta_{b}\right)=528.44 \mathrm{HP}$

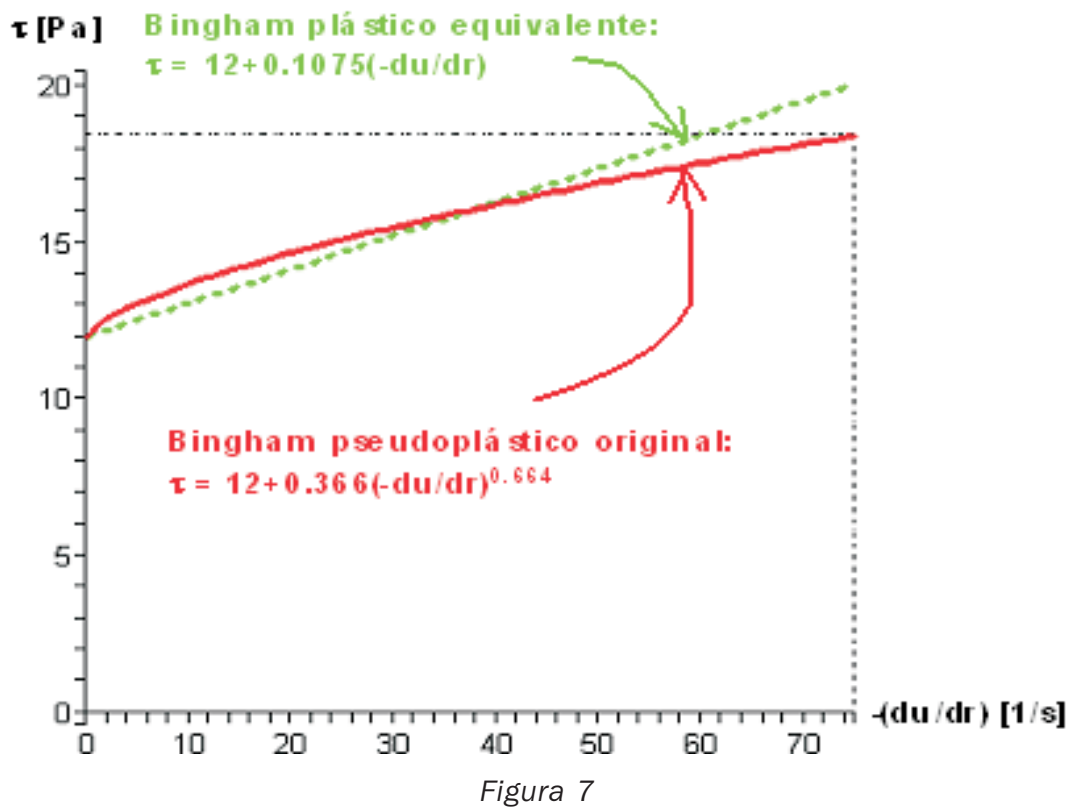




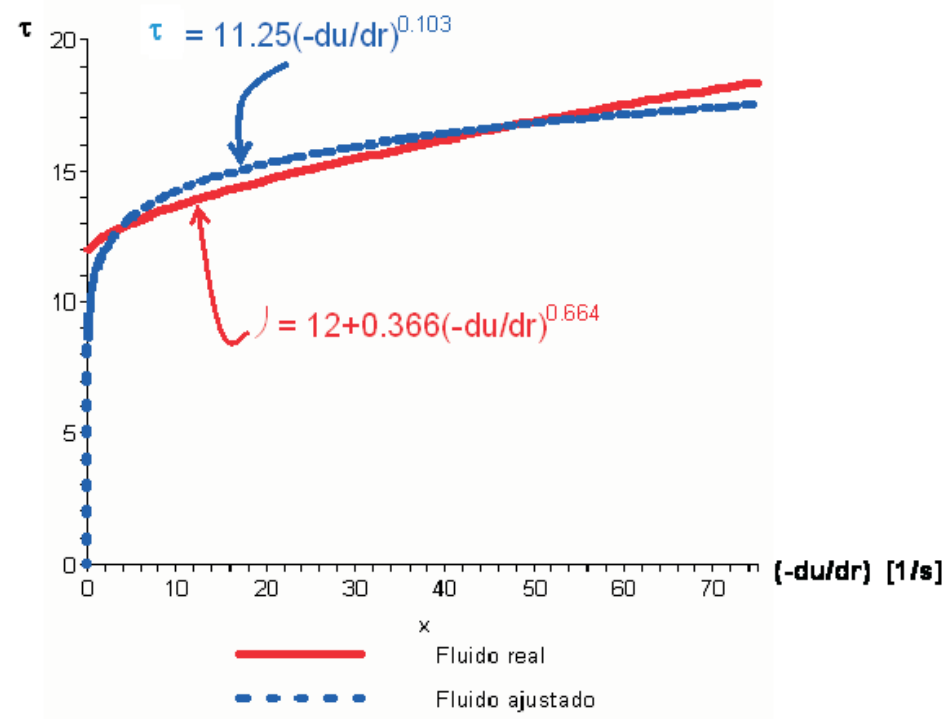

Figura 8

En el cuadro 2 se presenta un resumen de las potencias obtenidas con las dos propuestas.

\begin{tabular}{|c|c|}
\hline PROPUESTA & POTENCIA DE BOMBEO (HP) \\
\hline 1 & 524.45 \\
\hline 2 & 528.44 \\
\hline
\end{tabular}

Cuadro 2

Por considerarlo de interés, a continuación se presentan los resultados obtenidos con un fluido real de nuestro medio. No se indica con detalle el procedimiento seguido, porque sería una repetición del que se aplicó en el ejemplo anterior.

\section{Aplicación de las 2 propuestas al cálculo del bombeo para el lodo de la Planta de Tratamiento de Aguas Negras de Ciudad Universitaria de la UNAM}

En el Departamento de Alimentos y Biotecnología de la Facultad de Química de la UNAM, se realizó la caracterización del lodo residual de la Planta de Tratamiento de Aguas Negras de Ciudad Universi- taria. Se utilizó el reómetro de Formación Controlada ARES RFS III TA INSTR que se indica en la siguiente fotografía

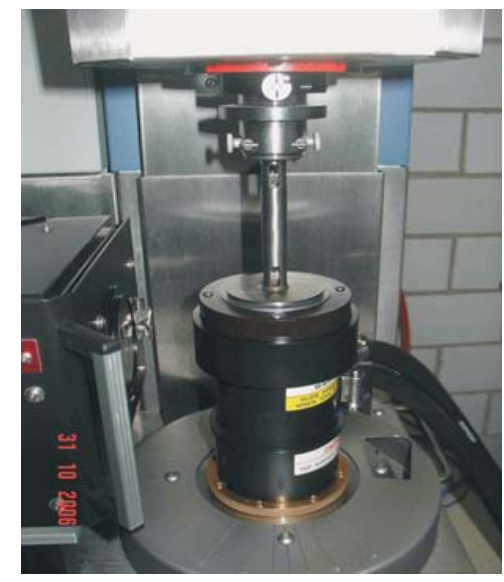

En la figura 9 se registran los parámetros obtenidos de la caracterización del lodo, tal como los presenta el monitor del reómetro mencionado.

Como puede verse, tanto en los parámetros obtenidos como en la curva reológica producida por el monitor del aparato, los resultados 
permitieron comprobar que efectivamente se trata de una sustancia tipo Bingham pseudoplástico, cuya ecuación es la siguiente:

$$
\tau=0.34507+1.26110(-\mathrm{du} / \mathrm{dr})^{0.22021}
$$

Con estos datos se hicieron los cálculos aplicando las dos propuestas mencionadas. Se utilizaron los mismos datos físicos del ejemplo señalado anteriormente $\mathrm{y}$, desde luego sólo se cambió el fluido.

Para la propuesta 1, el Bingham plástico equivalente resultó el descrito por la ecuación:

$$
\tau=0.34507+0.089425(-d u / d r)
$$

Y para la propuesta 2, la ecuación del pseudoplástico simple equivalente fue:

$$
\tau=1.5(-\mathrm{du} / \mathrm{dr}){ }^{0.205}
$$

Los resultados principales obtenidos se registran en el cuadro 3

\begin{tabular}{|c|c|}
\hline Propuesta & Potencia de Bombeo (HP) \\
\hline 1 & 206.06 \\
\hline 2 & 186.30 \\
\hline
\end{tabular}

Cuadro 3

\section{Conclusiones y recomendaciones}

- Los lodos residuales de las Plantas de Tratamiento de Aguas Negras son no newtonianos y específicamente fluidos tipo Bingham pseudoplástico.

- Debe procurarse trabajar siempre en régimen laminar porque el turbulento produce incrementos fuertes en las pérdidas por fricción.

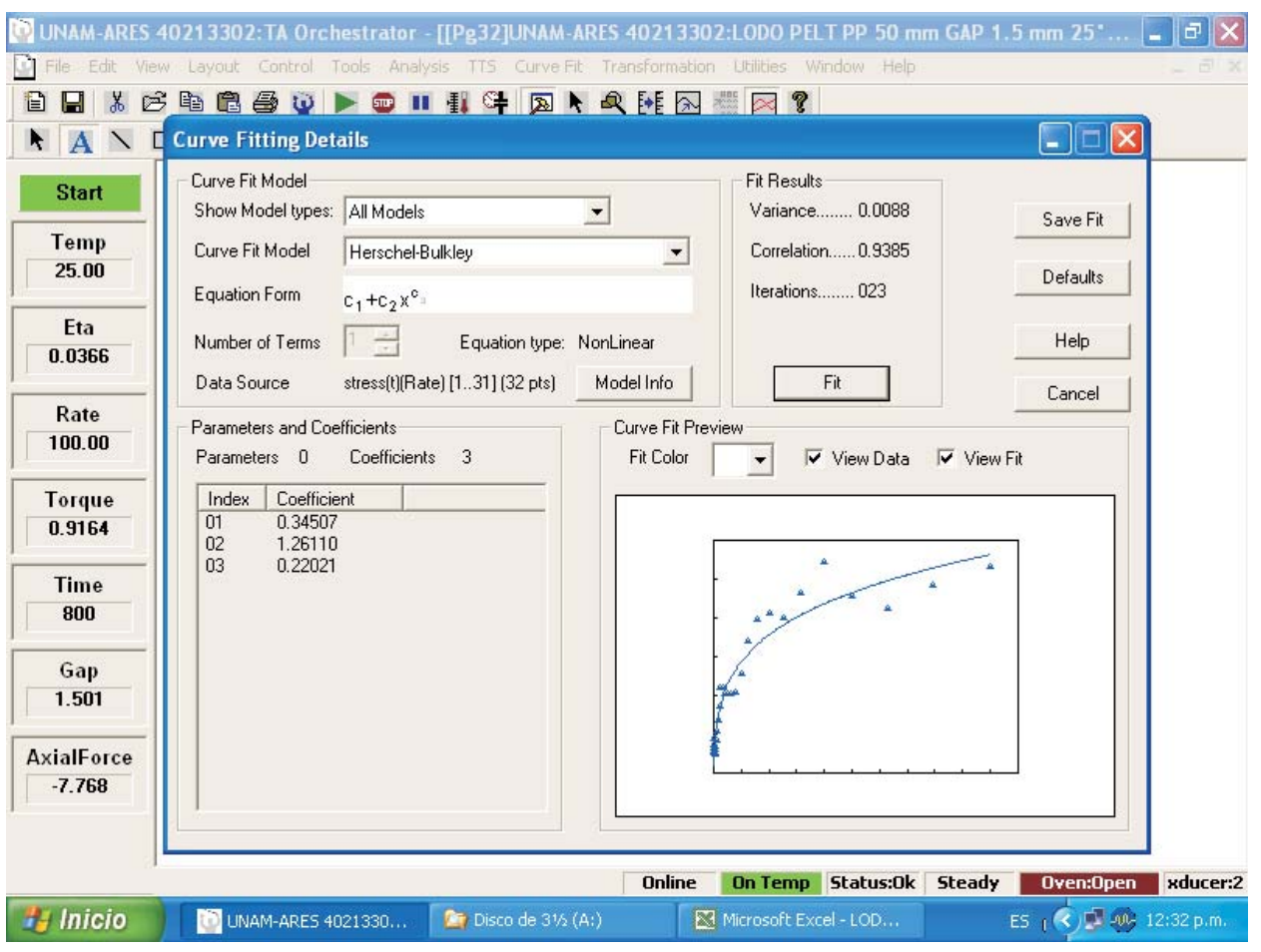

Figura 9 
- La zona más conveniente de régimen es la que se encuentra cercana al principio del flujo turbulento, pero aun dentro del régimen laminar .

- Como los parámetros de la ecuación reológica de cada sustancia no newtoniana tienen valores diferentes. Es indispensable siempre determinar en el laboratorio dichos parámetros (caracterizar el fluido).

- Se observa congruencia entre los resultados obtenidos con las dos Propuestas, por lo que se considera que son válidas.

\section{Referencias}

Honey H.C. and Pretorius W.A. Laminar floe pipe hydraulics of pseudoplastic-thixotropic swage sludges. Water SA [en línea]. Vol. 26. No. 1. January 2000. Disponible en: http://www.wrc. org.za
Lester C.B. Hydraulics for pipeliners. $2^{a}$ Edición. London, Paris. Gulf Publishing Company, 1994.

Levenspiel 0 . Engineering flow and heat exchange. NY, London, Plenium Press, 1986

Shook C.A. y Roco M.C. Slurry flow. Principles and practice. Boston. Butterworth-Heinemann, 1991.

Slatter P.T. The Rheological characterisation of sludges. IAWQ Journal. Wat. Sci. Tech, 36 (11): 9-18. 1997.

Slatter P.T. (2004), The hydraulic transportation of thickened sludges. Water Institute of South Africa (WISA), Biennial Conference. Cape Town, South Africa. Sludges in Straight Pipes. Water Research Centre. UK.

Slatter P.T. \& Wasp E.J. The laminar/turbulent transition in large pipes $\left(10^{\text {th }}, 2000\right)$. International Conference on Transport and Sedimentation of Solid Particles-Wroc ${ }^{3}$ aw: 4-7 September.

\section{Semblanza del autor}

Humberto Gardea-Villegas. Ingeniero civil. Maestro en hidráulica, candidato a doctor en hidráulica, en la Facultad de Ingeniería de la UNAM. Profesor en el área de hidráulica desde 1960 a la fecha. Becado durante 1962 y 1963 en el Instituto de Investigaciones Hidráulicas de Praga, Checoslovaquia. Semestres sabáticos en la Universidad Nacional de Colombia (1998) y en la Universidad Técnica Checa de Praga (2001). Viajes de estudio a la República Checa, Estados Unidos, Brasil, Venezuela, Colombia y Argentina. Actividades profesionales en la Secretaría de Recursos Hidráulicos, la Secretaría de Obras Públicas y la Comisión Federal de Electricidad. Consultor independiente en varias empresas privadas. Autor de cuatro libros: Uno sobre aprovechamientos hidroeléctricos, otro referente a canales y dos acerca de problemas de aplicación de los mismos temas. 